\title{
COMPUTER SIMULATION OF ELECTRON TRANSFER IN MOLECULAR ELECTRONIC DEVICES
}

\author{
Helena M. G. Correia, Marta M. D. Ramos* \\ Departamento de Física, Universidade do Minho, Campus de Gualtar, \\ 4710-057 Braga, Portugal
}

\begin{abstract}
The study of electron transfer through individual molecules bound to metal electrodes has become important due to the potential application in molecular electronic devices. Since the electronic and atomic motions in these molecules influence each other they need to be treated self-consistently. We have used self-consistent quantum chemistry molecular dynamics calculations to discuss some of the issues related to electron transfer through a spatially symmetric [9,10-Bis((2'-para-mercaptophenyl)-ethinyl)-anthracene] and an asymmetric [1,4$\operatorname{Bis}\left(\left(2^{\prime}\right.\right.$-para-mercaptophenyl)-ethinyl)-2-acetyl-amino-5-nitro-benzene] molecule bound to metal electrodes. Specifically addressed are the effects of voltage inversion on electron transfer between electrodes through both molecules. Our results show an electron transfer behaviour that reproduces the spatial symmetry of the molecules in agreement with experimental current-voltage data. The change in time of electron density and dimerisation at specific atomic sites is also discussed.
\end{abstract}

Keywords: Quantum modelling, electron transfer, molecular wires.

\section{Introduction}

Recent progress in atomic scale modelling (e.g. methods combining molecular dynamics with self-consistent quantum mechanical methods [1]) have greatly advanced our knowledge of electronic transport through electron-lattice coupled systems such as conjugated polymer chains [2, 3]. With the increasing interest of molecular electronic devices [4], it becomes

\footnotetext{
" Corresponding Author: Tel:+351 253 604330; Fax:+351 253678 981; E-mail: marta@ fisica.uminho.pt
} 
crucial to have a firm understanding of the nature of the charge carriers in a conjugated molecular wire.

Reichert et al [5] suggested that current-voltage characteristics of the metal-conjugated molecule-metal system reproduce the spatial symmetry of the molecules with respect to the direction of electron flow, when the connection to both electrodes is symmetrically realized by a chemical bond. However, these interpretations are not completely justified.

Despite the experimental evidence of lattice effects on electronic conduction through organic molecules [6], the lattice is frequently assumed rigid in calculations of tunnelling current through the molecular wires [7].

Since electronic and lattice motions influence each other in conjugated molecules, we used a self-consistent quantum chemistry molecular dynamics method to investigate the electron transfer and charge storage in spatially symmetric and asymmetric, but otherwise similar, conjugated molecules as a function of the applied electric field between both electrodes. Results of these calculations will be used to assess the consequences for the interpretation of Reichert et al. [5] experiments. This dynamical approach is also used to calculate the time evolution of both electron density and lattice distortion at specific atomic sites, which will help us to discuss the validity of rigid lattice assumption for charge transport calculations in conjugated molecules with strong electron-phonon coupling.

\section{Theoretical method}

To study the electron transfer from the cathode to the anode through a conjugated molecule sandwiched between both electrodes, we performed self-consistent calculations of both electronic structure and molecular geometry of the metal-molecule-metal system, using the

CHEMOS code $[1,8]$. In this code an approximate molecular orbital method called CNDO (Complete Neglect of Differential Overlap), with a set of parameters selected to give the best 
overall fit to minimal basis set Hartree-Fock calculations [9], is used to calculate the electronic structure of the entire cluster. Differentiating the self-consistent total internal energy with respect to the nuclear coordinates leads to the atomic forces. Once these forces are known, a dynamic simulation of the atomic relaxation can be accomplished using the molecular dynamics method.

In the work reported here, the chemical interactions between the atoms of the molecule and between those and the metal atoms of both electrodes bound to the molecule are considered. The same approach was used in our earlier work [10-12] for trans-polyacetylene and polyimide in contact with metal atoms.

\section{Results and discussion}

Before any electric field was applied, a spatially symmetric [9,10-Bis $\left(\left(2^{\prime}\right.\right.$-paramercaptophenyl)-ethinyl)-anthracene] and an asymmetric [1,4-Bis((2'-para-mercaptophenyl)ethinyl)-2-acetyl-amino-5-nitro-benzene] molecule bound to sodium atoms of the electrodes (figure 1) were relaxed to equilibrium.

When an uniform electric field is applied to both molecules along the sulfur-to-sulfur molecular axis, the metal atoms bound to the sulfur atoms of both molecules are taken to be fix. The chemical interaction between the conjugated molecules and the metal atoms of both electrodes enables us to capture qualitative features of electron transfer from the cathode to the anode through those molecules without using any scattering method. Our approach can be easily applied to other metals and molecules.

Figure 2 shows the changes in the charge of the symmetric molecule and both metal atoms caused by the applied electric field. Our results suggest that when electrons flow from the metal atom of the cathode to the metal atom of the anode through the molecule, charge is stored in the molecule. The amount of charge stored in this molecule changes nonlinearly with the field strength. The sign of the charge stored in the symmetric molecule is independent of 
voltage inversion. These results may explain the symmetry observation in the experimental current-voltage (I-V) characteristics of the symmetric molecule [5] with symmetric contact realization.

To understand what really changes when there is electron transfer between cathode and anode through the asymmetric molecule, we examine the changes in charge of metal atoms of both electrodes and of the asymmetric molecule for different applied electric fields along the molecular axis. The results presented in figure 3 show two main features. First, the is charge stored in the asymmetric molecule varies linearly with the applied bias voltage. Second, the charge stored in this molecule changes sign with voltage inversion. These results could be a plausible explanation for the asymmetric current-voltage characteristics with respect to the direction of current flow observed in the experiments [5].

For both symmetric and asymmetric molecules, the electron transfer between the metal atoms of the cathode and the anode occurs before the relaxation of the atomic positions in the molecule takes place.

Because of the electron-lattice coupling in these conjugated molecules, the electron transfer described above leads to a lattice motion away from its equilibrium configuration. The response of the conjugated molecule to the electron transfer can be studied by calculating the dimerization parameter defined in reference [8] by:

$$
d_{n}=(-1)^{n}\left(b_{n, n+1}-b_{n-1, n}\right)
$$

where $b_{n, n+1}$ is the length of the bond joining carbon atoms $n$ and $n+l$ of the molecular backbone.

The induced lattice vibrations are shown in figure 4. As a result there is a periodic change in the atomic charge of all the atoms of the molecule (figure 5). The changes in time of both atomic charges and lattice vibrations have similar frequencies. The electronic and lattice time evolution of the molecule leads also to a periodic change in the charge of the metal atoms 
bound to the molecule. The amplitude of the changes predicted for the metal atoms does not seem to be affected by the strength of the applied electric field (figure 6).

Figures 7 and 8 show the change in charge distribution among the carbon atoms of the molecular backbone and the change in dimerization at the corresponding atomic sites for different times. Because of the electron-lattice coupling, the dimerization pattern of the molecule change shape and seems to move through it to some extent while electron density oscillates in time.

\section{Conclusions}

The results presented in this paper illustrate the complex processes involved in electron transfer across metal/conjugated molecule/metal structures. We show that a significant difference in electron transfer through symmetric and asymmetric molecules is due to the charge storage in the molecule. The change in sign of the stored charge in the asymmetric

molecule when the voltage is reversed can be a plausible explanation for the asymmetric current-voltage characteristics obtained in the experiments.

Besides, electron transfer through conjugated molecules leads to atomic vibrations along the molecular axis. Because of the strong coupling between electrons and atomic vibrations in these molecules, the lattice distortion induces a periodic change in the atomic charge of the molecular wire. Both effects are expected to modify the charge transport through conjugated molecules, therefore the formulation of the transport in these molecules needs a self-consistent solution for both electronic and lattice time evolutions.

\section{Acknowledgement}

This work is part of the research project POCTI/CTM/41574/2001, approved by the Portuguese Foundation for Science and Technology (FCT) and POCTI and supported by the 
European Community Fund FEDER. One of us (H.M.G.C.) is also indebted to FCT for financial support under PhD grant No. SFRH/BD/11231/2002.

\section{References}

[1] D. S. Wallace, A. M. Stoneham, W. Hayes, A. J. Fisher, A. H. Harker and J. Phys. Condens. Matter, 3 (1991) 3879.

[2] A. M. Stoneham, M. M. D. Ramos, A. M. Almeida, H. G. Correia, R. M. Ribeiro, H. Ness and A. J. Fisher, J. Phys. Condens. Matter, 14 (2002) 9877.

[3] H. M. G. Correia and M. M. D. Ramos, Materials Science and Engineering C 23 (2003) 773.

[4] C. Joachim, J. K. Gimzewski and A. Aviram, Nature, 408 (2000) 541.

[5] J. Reichert, R. Ochs, D. Beckmann, H. B. Weber, M. Mayor and H. V. Lohneysen, Phys. Rev. Lett., 88 (2002) 176804.

[6] A. Kadyshevitch and R. Naaman, Phys. Rev. Lett., 74 (1995) 3443.

[7] M. P. Samanta, W. Tian, S. Datta, J. I. Henderson and C. P. Kubiak, Phys. Rev. B 53 (1996) R7626.

[8] D. S. Wallace, D. Phil. Thesis, University of Oxford, 1989.

[9] J. A. Pople and D. L. Beveridge, “Approximate Molecular Orbital Theory”, McGraw-Hill, New York (1970).

[10] M. M. D. Ramos, A. M. Stoneham and A. P. Sutton, Acta Metall. Mater., 41 (1993) 2105.

[11] M. M. D. Ramos and J. P. P Almeida, Computational Materials Science 10 (1998) 184.

[12] M. M. D. Ramos and J. P. P. Almeida, Journal of Materials Processing Tecnology, 92-93 (1999) 147. 


\section{List of figure captions:}

Figure 1 - Schematic diagram of: a) [9,10-Bis((2'-para-mercaptophenyl)-ethinyl)-anthracene] and b) [1,4-Bis((2'-para-mercaptophenyl)-ethinyl)-2-acetyl-amino-5-nitro-benzene] molecules, are chemically bound to sodium atoms of both electrodes. Some atoms are numbered for easier identification.

Figure 2 - Change in the charge of [9,10-Bis((2'-para-mercaptophenyl)-ethinyl)-anthracene] molecule and metal atoms of both electrodes bound to the molecule when an electric field is applied along to molecular axis. The lines are a guide to the eye.

Figure 3 - Change in the charge of [1,4-Bis((2'-para-mercaptophenyl)-ethinyl)-2-acetylamino-5-nitro-benzene] molecule and metal atoms of both electrodes bound to the molecule when an electric field is applied along to molecular axis. The lines are a guide to the eye.

Figure 4 - Change of dimerization as a function of time at selected atomic sites indicated in figure 1a), when an electric field of $5 \times 10^{8} \mathrm{~V} / \mathrm{m}$ is applied along the molecular axis.

Figure 5 - Change of atomic charges as a function of time for selected atoms of $\left[9,10-\operatorname{Bis}\left(\left(2^{\prime}-\right.\right.\right.$ para-mercaptophenyl)-ethinyl)-anthracene] molecule indicated in figure 1a), when an electric field of $5 \times 10^{8} \mathrm{~V} / \mathrm{m}$ is applied along the molecular axis.

Figure 6 - Change of atomic charges as a function of time for the metal atoms of both electrodes bound to the molecule indicated in figure 1a), when different electric fields are applied along the molecular axis. 
Figure 7 - Charge storage in carbon atoms of the molecular backbone shown in figure 1a) for three different times. The marks on the when an electric field of $5 \times 10^{8} \mathrm{~V} / \mathrm{m}$ is applied along the molecular axis. The lines are a guide to the eye.

Figure 8 - Dimerization pattern of the molecular backbone shown in figure 1a) for three different times. The marks on the when an electric field of $5 \times 10^{8} \mathrm{~V} / \mathrm{m}$ is applied along the molecular axis. The lines are a guide to the eye. 
Figure 1

a)

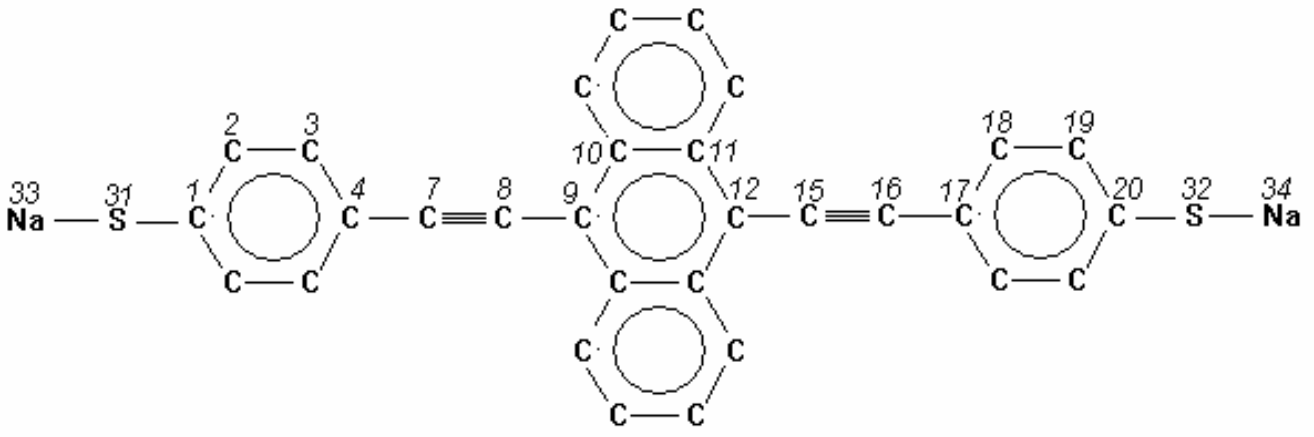

b)

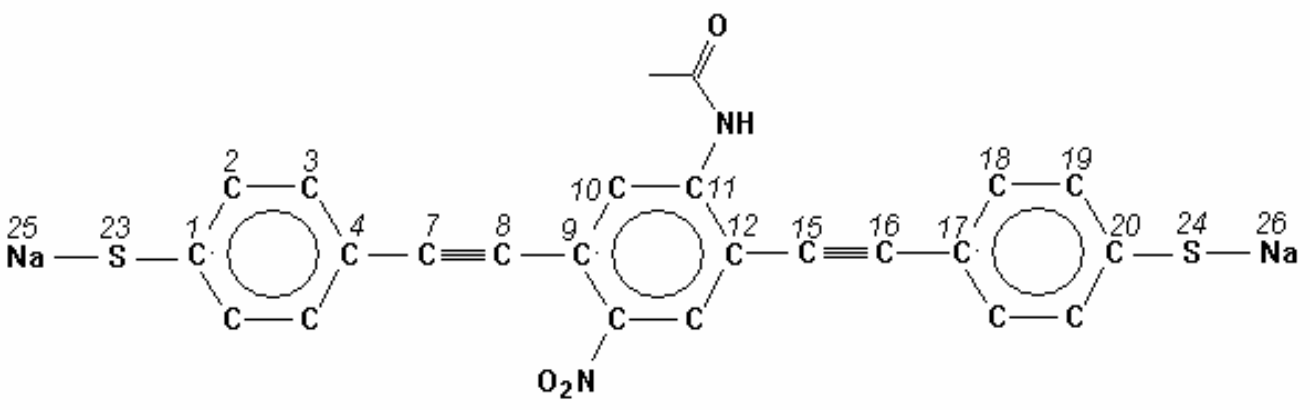


Figure 2

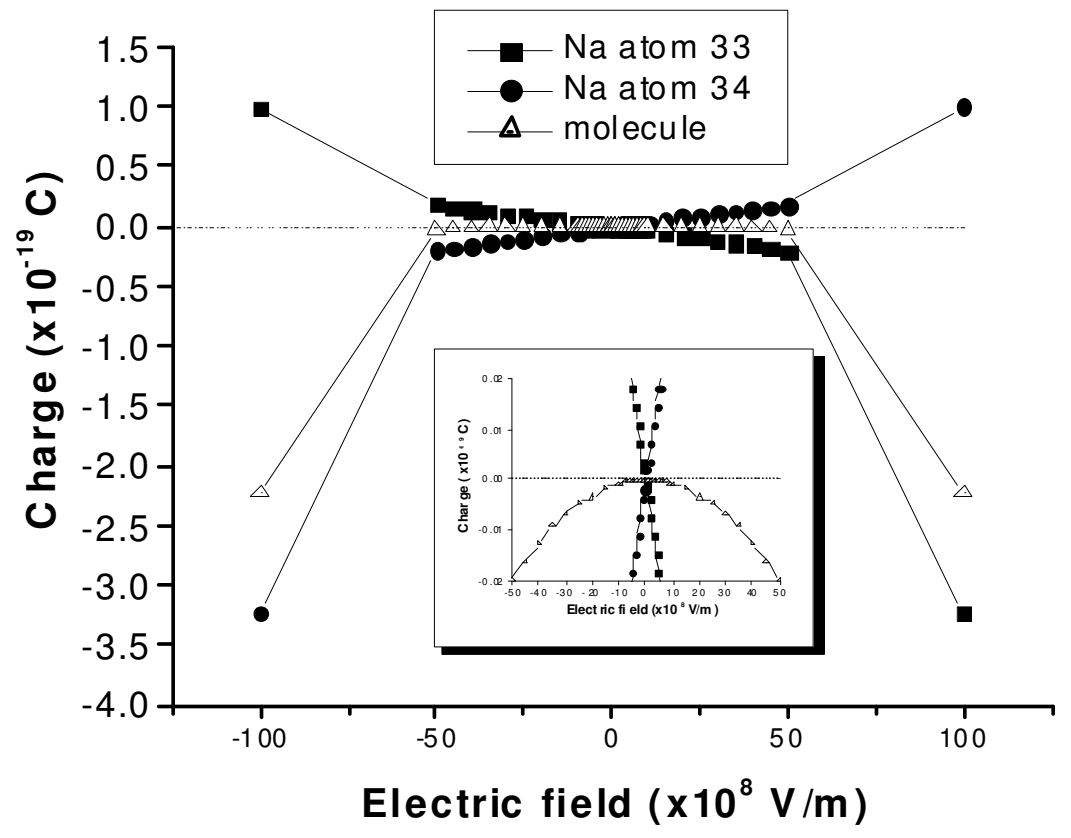


Figure 3

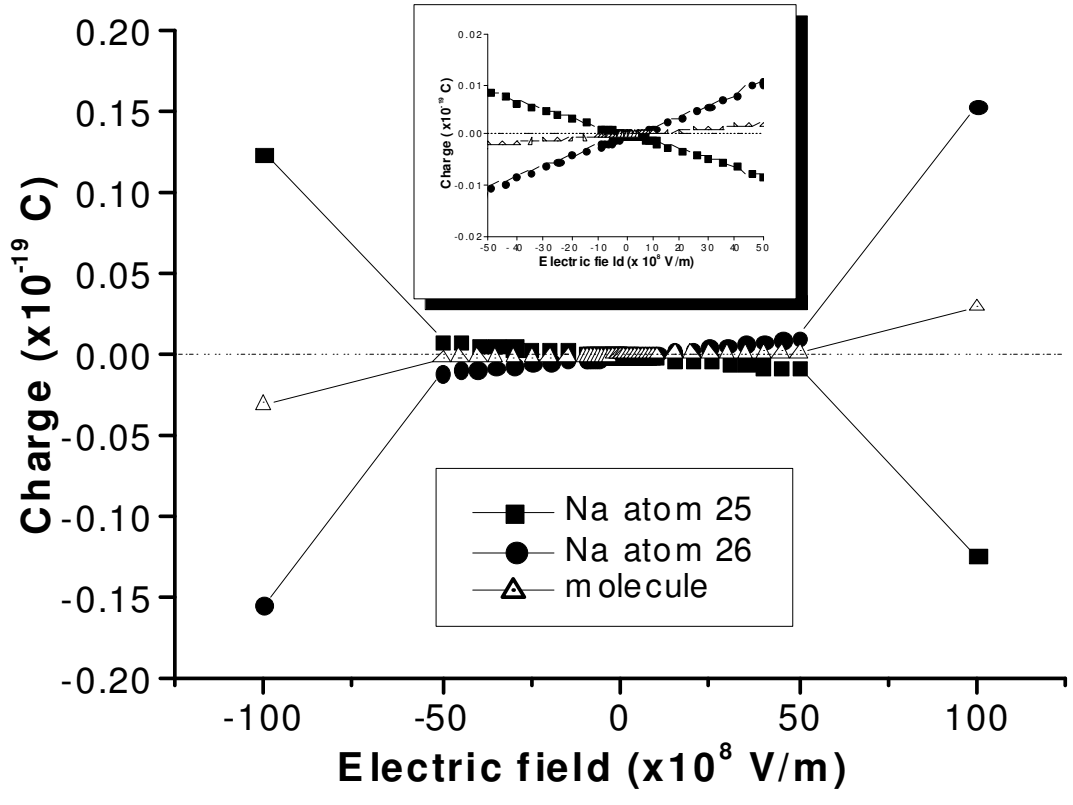


Figure 4

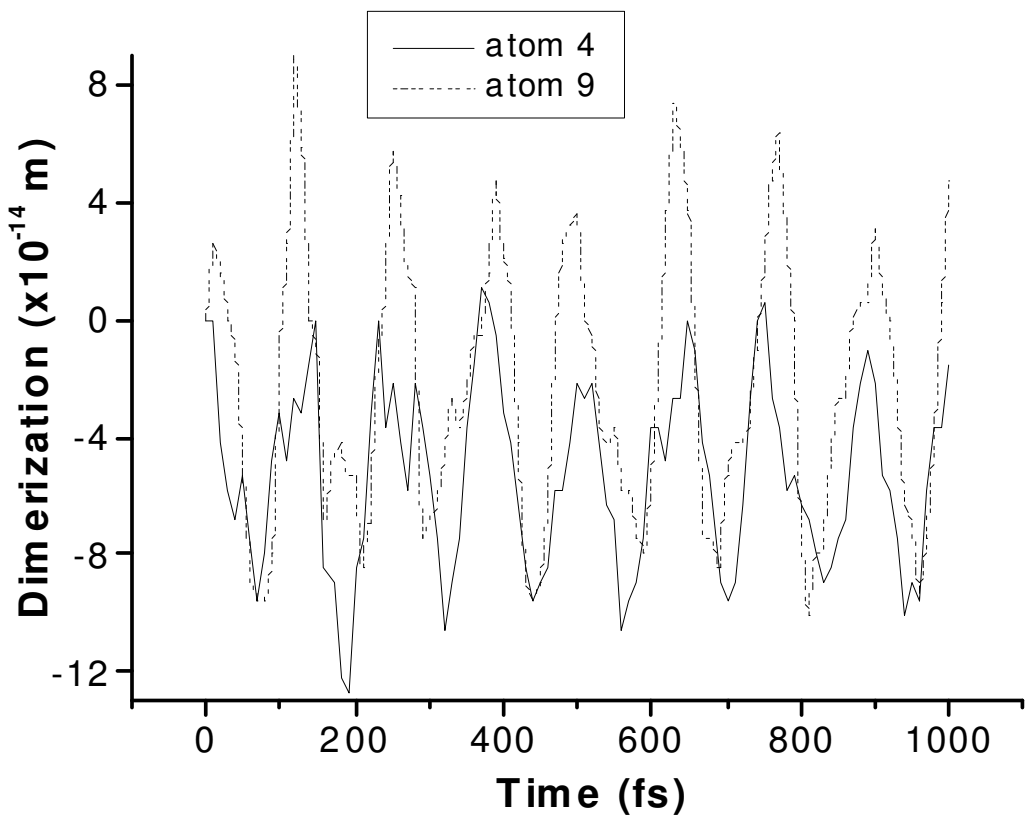


Figure 5

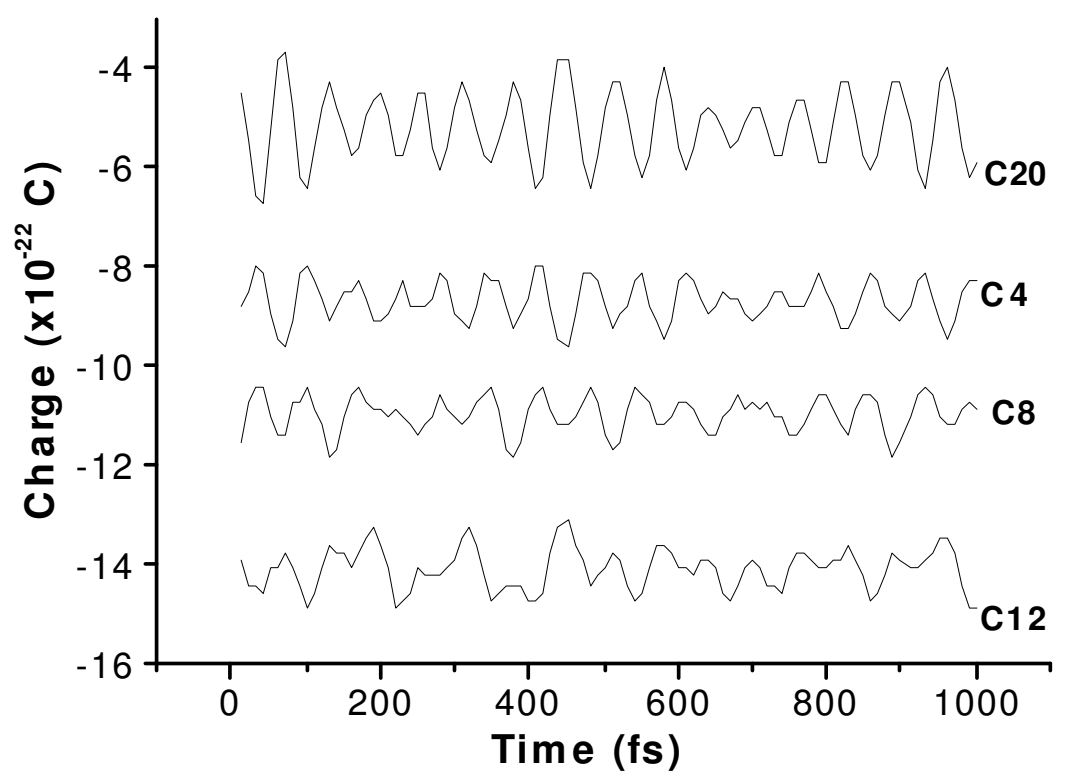


Figure 6

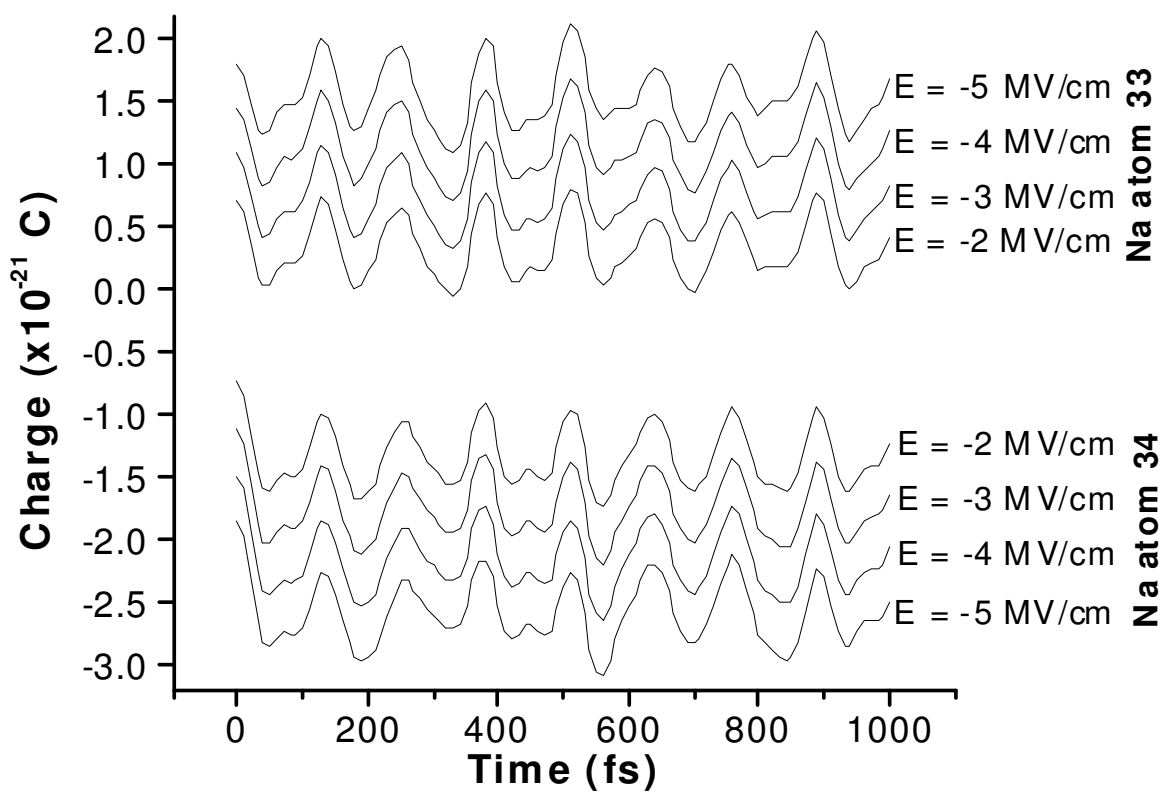


Figure 7

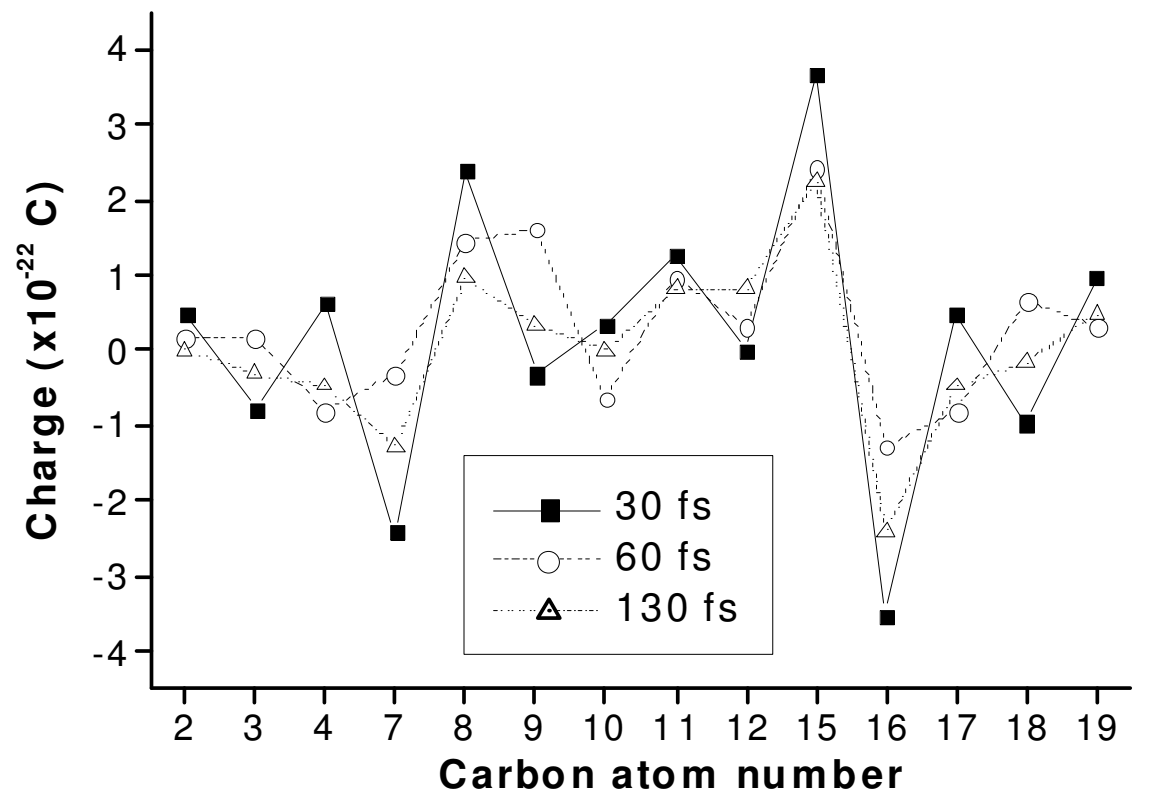


Figure 8

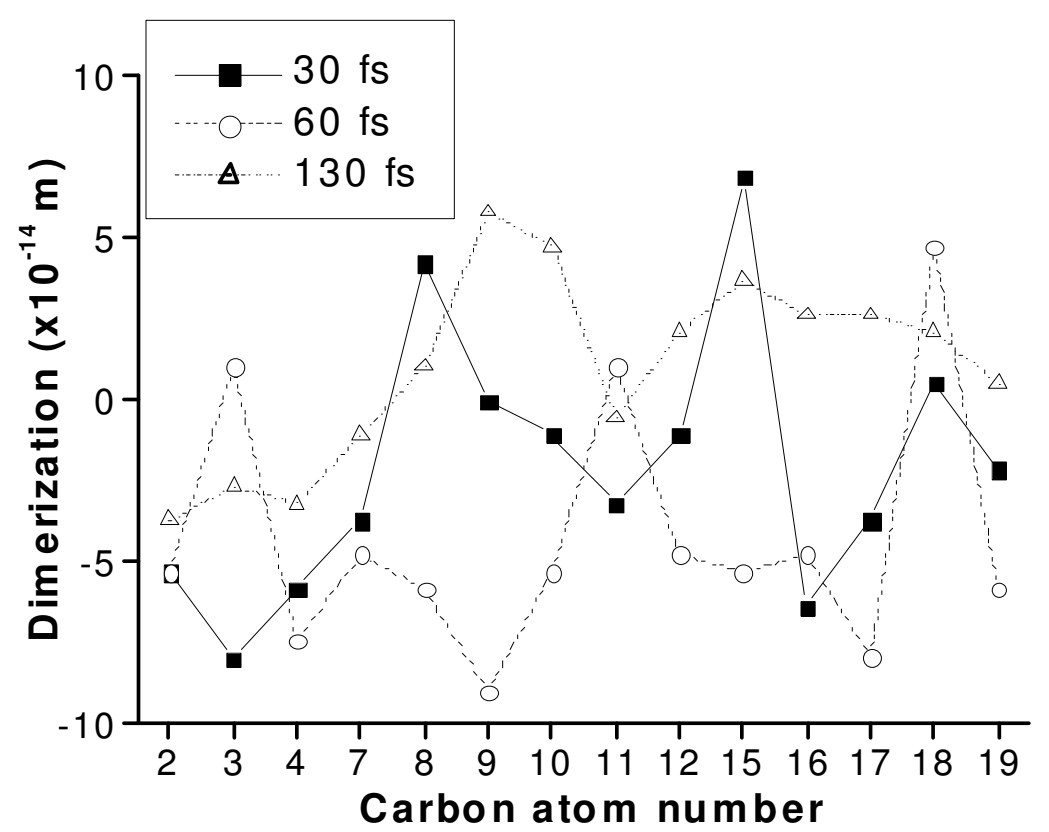

